AperTO - Archivio Istituzionale Open Access dell'Università di Torino

\title{
Associations between dogs that were serologically positive for Rickettsia conorii relative to the residences of two human cases of Mediterranean spotted fever in Piemonte (Italy)
}

\section{This is the author's manuscript}

Original Citation:

Availability:

This version is available http://hdl.handle.net/2318/7721 since 2016-11-29T17:08:07Z

Publisher:

Harcourt Publishers Limited:Foots Cray High Street, Sidcup Kent DA14 5HP United Kingdom:011 4420

Published version:

DOI:10.1016/S0167-5877(03)00079-5

Terms of use:

Open Access

Anyone can freely access the full text of works made available as "Open Access". Works made available under a Creative Commons license can be used according to the terms and conditions of said license. Use of all other works requires consent of the right holder (author or publisher) if not exempted from copyright protection by the applicable law. 


\title{
Associations between dogs that were serologically positive for Rickettsia conorii relative to the residences of two human cases of Mediterranean spotted fever in Piemonte (Italy)
}

\author{
A. Mannelli ${ }^{\mathrm{a}, *}$, M.L. Mandola ${ }^{\mathrm{b}}$, P. Pedri ${ }^{\mathrm{c}}$, \\ M. Tripoli ${ }^{\text {a }}$, P. Nebbia ${ }^{\text {a }}$ \\ a Dipartimento di Produzioni Animali, Epidemiologia ed Ecologia, \\ Via Leonardo da Vinci 44, 10095 Grugliasco (To), Italy \\ b Istituto Zooprofilattico Sperimentale del Piemonte, Liguria e Valle D'Aosta, \\ Via Bologna 148, 10154 Torino, Italy \\ ${ }^{\mathrm{c}}$ Azienda Sanitaria Locale 15, Cuneo, Italy
}

\begin{abstract}
A geographic information system and $K$-function analysis were used to evaluate the spatial association of canine serological results for Rickettsia conorii, the causative agent of Mediterranean spotted fever (MSF), and clinical cases of MSF in humans in Piemonte, northwest Italy. The residences of dog owners were clustered in two rural villages in the province of Cuneo, where two human cases of MSF occurred in 1997 and 1998. Eighteen out of 116 dogs examined were positive by indirect immunofluorescent assay (IFA + , titre $\geq 1: 160$ ) for MSF. $K$-functions were compared for IFA + dogs and for all dogs sampled. Monte Carlo and bootstrap simulations demonstrated that clustering of IFA+ dogs was significantly greater than clustering of all dogs, at distances of less than $0.6 \mathrm{~km}$ from human cases of MSF. Logistic regression analysis indicated that the risk of being IFA+ was highest for dogs residing within the first quartile of distance $(0.7 \mathrm{~km})$ from human cases of the disease, and for dogs that were not confined. However, year and season of blood collection were not associated with IFA status. It was concluded that a relatively high dog population density along with a rural or semi-rural environment favours the occurrence of emergent foci of MSF in the province of Cuneo. (C) 2003 Elsevier Science B.V. All rights reserved.
\end{abstract}

Keywords: Mediterranean spotted fever; Dogs; Serology; Spatial analysis; GIS

\footnotetext{
* Corresponding author. Tel.: +39-01167-09186; fax: +39-01123-69186.

E-mail address: alessandro.manneli@unito.it (A. Mannelli).
} 


\section{Introduction}

Mediterranean spotted fever (MSF) is a tick-borne zoonosis which usually presents as a mild illness that can go clinically unnoticed. However, severe forms of MSF may occur, which are characterised by high fever with renal and cardiac involvement that may cause mortality, particularly in older patients (Raoult and Roux, 1997). Rickettsia conorii, the causative agent of MSF, is maintained in the Mediterranean area through transovarial and transstadial transmission within populations of the brown dog tick, Rhipicephalus sanguineus. Vertebrate hosts are essential in providing blood meals for ticks, but their role in the transmission cycle of $R$. conorii is unclear. Adult $R$. sanguineus show a high degree of affinity for dogs but immature stages are considered to be less host-specific, and are probably responsible for the transmission of the infection to humans, particularly during summer months (Maroli et al., 1996).

Rickettsioses are considered as paradigms of emerging infections due to their increasing incidence and expanding geographical ranges. Changes in frequency and spatial distribution of tick-borne zoonoses are affected by natural and man-made associated factors. The emergence of MSF, for example, has been associated with higher than usual temperatures and lower rainfall in Spain, and with a decrease in the number of days of frost in France (Raoult et al., 1992; Raoult and Roux, 1997). In Italy, the disease is most often reported in southern regions (Sicily and Latium), but it may also occur in central and northern regions (Maroli et al., 1996). In Piemonte (northwest Italy), MSF was first reported during the 1970s in people that travelled to other regions, whereas the first autochthonus cases were reported in the mid 1980s.

Surveillance is necessary to detect new areas at risk of MSF, to identify risk factors, and to direct preventive measures. Valid risk indicators are essential for these purposes. Human cases of MSF cannot be considered as a sensitive indicator because clinical disease is relatively rare and there is a lack of consistent diagnostic criteria. On the other hand, the detection of antibodies against $R$. conorii in dogs has been used in epidemiological studies due to the high levels of exposure to $R$. sanguineus and to the intense antibody response to the organism encountered in dogs (Keysary et al., 1988; Delgado and Carmenes, 1995; Segura-Porta et al., 1998). Masoero et al. (1991) found that $35.5 \%$ of dogs sampled in the proximity of residences of human MSF cases in the province of Cuneo (Piemonte) were seropositive (cut-off titre $\geq$ 1:40). The objective of the present study was to optimise the use of canine serology as a risk indicator of MSF in humans, through the application of a geographic information system (GIS) to study the spatial association between seropositive dogs and human cases of MSF.

\section{Materials and methods}

\subsection{Human cases of MSF in Piemonte}

Information on human cases of MSF that were notified in Piemonte between 1997 and 1999 were obtained from the website of the regional service of epidemiology. ${ }^{1}$ Diagnosis of

\footnotetext{
${ }^{1}$ SSEPI, http://www.asl20.piemonte.it/SEPI/sorveglianza_mi.html.
} 
MSF is normally based upon clinical symptoms and a positive indirect immunofluorescent assay (IFA). At-risk population data were obtained from a demographic database of the region. ${ }^{2}$ These data were used to calculate incidence rate approximations (number of cases per million inhabitants per year) for each province of Piemonte, using the EPITABLE program in EPINFO, Version 6.04 (Dean et al., 1995). Probabilities of the observed numbers of cases, under the null hypothesis of a random (Poisson) distribution of cases within the provinces, were calculated using EPITABLE.

\subsection{Collection of serum samples from dogs in the province of Cuneo}

Serum samples were collected from dogs by public veterinarians, from October 1997 to September 1999, in four municipalities of the province of Cuneo, Piemonte (Morozzo, Margarita, Castelletto Stura, Montanera; overall study area $=61.1 \mathrm{~km}^{2}$ ). In this study area, two people developed MSF in autumn 1997 and in spring 1998. It was possible to ascertain that they had been bitten by ticks at their residences in the villages of Morozzo and Margarita (personal communication provided by Health District 15, Piemonte). Sera were collected from dogs during four sessions of mandatory tattooing. However, compliance with tattooing may not be geographically uniform in the study area. In fact, dogs living in villages are considered more likely to undergo tattooing than those living in the countryside. For this reason, other canine sera were collected during routine veterinary visits to farms. At the time of blood collection, a questionnaire was completed based on information provided by the owner of the dog. Data on breed of dog, sex, age, hair colour, hair length were collected along with information on the potential for exposure to ticks such as type of residence area (urban, suburban, rural), use of dog, confinement (kept in house, kept in yard, chained, free), presence of other animals, detection of ticks at the time of blood collection or previously detected by owners, previous treatment against ectoparasites or recent administration of drugs for other reasons and clinical signs of disease. Sites of residence of dogs that were bled during tattooing were recorded on a map, whereas co-ordinates of farms were obtained using a geographical positioning system $\left(\right.$ Garmin $^{\circledR}$, GPS 38). Dog sera were tested for antibodies against $R$. conorii, at the Istituto Zooprofilattico Sperimentale of Piemonte, Liguria, e Valle d'Aosta, using an IFA (IFA, Biomerieux ${ }^{\circledR}$ ). In order to reduce the probability of false positive results that could be due to cross-reactions with antigens other than that of $R$. conorii (e.g. non-pathogenic rickettsiae), dogs with an antibody titre of $\geq 1: 160$ were considered as positive (La Scola and Raoult, 1997).

\subsection{Spatial analysis}

Dog residences were digitised as points on a map of Piemonte (UTM co-ordinate system, zone 32), in a GIS (Atlas-GIS ${ }^{\circledR}$, Strategic Mapping Inc., Santa Clara, CA). Questionnaire data and serology results were entered into the GIS as attributes. In order to study the spatial association between canine serological results and risk of MSF in humans, a modified $K$-function (second order neighbourhood analysis) was applied to the data-point locations of all dogs sampled and of seropositive dogs, relative to residence sites of the two human cases

\footnotetext{
${ }^{2}$ BDDE, http://www.regione.piemonte.it/stat/bdde/index.htm.
} 
of MSF in Morozzo and Margarita. In general, second order analysis tests the randomness of spatial patterns by comparing the observed number of the total possible pairs of points that are within a specified distance of each other, with the number that would be expected in a random spatial distribution. Distances where clustering occurs and where departure from randomness is most pronounced can then be identified (Getis and Franklin, 1987; Boots and Getis, 1998; O'Brien et al., 1999). Second order neighbourhood analysis was modified by Kitron et al. (1992) to verify the higher degree of aggregation of tick-infested white-tailed deer around a known focus of Lyme borreliosis in northwest Illinois. In a similar approach, second order properties of the distributions of all dogs (IFA+ and IFA-) was characterised by modifying the equation for $K$-function as reported in Fotheringham et al. (2000), to test clustering of dogs around two points (i.e. the two human cases of MSF):

$$
\hat{K}(d)=\frac{|A|}{2 n} \sum_{i=1}^{2} \frac{N\left(C\left(x_{i}, d\right)\right)}{\alpha_{i}},
$$

where $d$ is the distance of dogs' residences from human cases of MSF, $A$ the study area, $n$ the number of dogs, $N$ the count of points (dogs' residence sites) within the circular region $C$ of radius $d$, around event $x_{i}$ (human MSF case residence site), and $\alpha_{i}$ is the correction factor for irregular boundaries of the study area. Such a correction is needed because the calculation of $\hat{K}(d)$ is based on the assumption that a point process is continuous beyond the boundaries of the study area. Such an assumption can be satisfied by weighting points whose distance from another point is greater than the distance to the border of the study area. The weight will increase $N$ to take into account the "missing" points, outside the study area. In this study, it was accomplished by including, as $\alpha_{i}$, the ratio between the area of the circle of radius $d$, to the fraction of the same area falling within the study area (Boots and Getis, 1998). To approximate the correction for each distance step, areas were measured by Atlas-GIS ${ }^{\circledR}$ software, for 10 distance bands, $1 \mathrm{~km}$ wide, around each human cases of MSF (Anon., 1990).

Eq. (1) was applied to the distribution of all dogs tested, and subsequently to IFA+ dogs, after exporting distance data from GIS to SAS ${ }^{\circledR}$, Version 8 (SAS Institute Inc., Cary, NC). The observed distance vector was used to fix increments to calculate $\hat{K}(d)$ (Boots and Getis, 1998). In order to test for clustering of dogs with regards to MSF cases, the estimated $K$-function from the sample of dogs was compared with the theoretical $K$-function for a process with uniform intensity corresponding to $\pi d^{2}$ (Fotheringham et al., 2000). If $\hat{K}(d)$ was greater than $\pi d^{2}$ at a given distance, then there were more dogs residing near MSF cases than it would have been expected by chance alone. In order to compare estimated and theoretical $K$-functions, the following expression was plotted, which equates to zero when the two functions are equal:

$$
\hat{l}(d)=\frac{1}{2} \log \left(\frac{\hat{K}(d)}{\pi}\right)-\log (d) .
$$

Natural log transformation was carried out to improve visual inspection of results. Monte Carlo simulation was implemented in order to test the null hypothesis of a random distribution of dogs with regards to human cases of MSF (Manly, 1991; Fotheringham et al., 
2000). The $\mathrm{SAS}^{\circledR}$ macro facility was used to generate statements and commands for multiple simulated data sets (SAS, 1990a,b). One hundred sets of random points, comprised within the range of co-ordinates of the observed dog residences, were generated using the RANUNI function. Points falling outside the boundaries of the study area were discarded by fixing limits for the $X$ co-ordinate values at different $Y$ value steps. A sample of points, corresponding to the number of examined dogs was drawn from each data set. Distances of selected points from human MSF cases were obtained using the Pythagorean theorem. $\hat{K}(d)$ was calculated for each distance step, taking into account GIS-generated border corrections, and $99 \%$ confidence interval $(\mathrm{CI})$ of $\hat{l}(d)$ were plotted with the observed results. Departure from a random distribution of dogs around human cases of MSF was considered as statistically significant (at the $1 \%$ level) at those distances where observed values of $\hat{l}(d)$ were greater than the 99th percentile of values obtained in the simulations (indicating clustering) or smaller than the 1st percentile of simulations (indicating longer distances than expected between dogs and human MSF cases). To compare the distribution of IFA + dogs with that of all dogs sampled, the corresponding $K$-functions were compared by a method described by Fotheringham et al. (2000), that consists of a modification of Eq. (2):

$$
\tilde{l}(d)=\log \left(\hat{K}_{\mathrm{IFA}+}(d)\right)-\log \left(\hat{K}_{\mathrm{all} \mathrm{dogs}}(d)\right),
$$

where $\hat{K}_{\mathrm{IFA}+}(d)$ and $\hat{K}_{\text {all dogs }}(d)$ were $K$-functions for IFA+ and all dogs sampled, respectively. When $\tilde{l}(d)=0$, there was no difference in clustering between IFA + dogs and all dogs sampled. To test the null hypothesis that IFA+ dogs were a random sample of all dogs sampled, two simulation approaches were used. Firstly, 100 random permutations of labels (using IFA + and IFA - as a binary labelling variable) were drawn and the observed values were compared with the 1st and 99th percentiles of simulated $\tilde{l}(d)$ at the corresponding distances. Using another simulation-based approach, 99\% CI for $\tilde{l}(d)$ were calculated using a bootstrap method. For this purpose, 100 data sets of IFA+ dogs were generated by sampling with replacement from the observations, using the macro language in SAS ${ }^{\circledR}$. The 1 st and 99th percentiles of the simulated distribution of $\tilde{l}(d)$ were obtained for each distance step and plotted against the observed results.

\subsection{Analysis of risk factors from questionnaire data}

Percentages of IFA+ dogs for each exposure level of categorical variables were obtained using the FREQ procedure in SAS ${ }^{\circledR}$. Median and first (Q1) and third (Q3) quartiles for age and for the distance of dogs' residence sites from the nearest human MSF case were obtained, for IFA + and IFA - subjects, using the UNIVARIATE procedure (SAS, 1990a). Based on the preliminary analysis, questionnaire variables were recoded as binary variables that were then used in two-by-two table analyses (PROC FREQ) to test their associations with dogs' IFA status. Variables where the $\chi^{2}$ test statistic was $P<0.25$ were subsequently included in a multiple logistic regression (unconditional maximum likelihood method; SAS, 1996) to test their adjusted effect on dog IFA status. The likelihood ratio test was used to assess the overall significance of the model (two-tailed significance level $\alpha=0.05$ ). The significance of each term in the model was tested by Wald's $\chi^{2}$. Estimated odds ratios (OR) and $95 \%$ Wald's CI were obtained as measures of predictor effects. Goodness-of-fit was 
tested by the Hosmer-Lemeshow test (Hosmer and Lemeshow, 1989). Where the effect of predictors was significant at the $25 \%$ level in the first logistic regression model, these were then included in a final model. Interactions between variables in the logistic regression models were not tested because of low sample sizes in each outcome group (Hosmer and Lemeshow, 1989).

\section{Results}

\subsection{Human cases of MSF in Piemonte}

In Piemonte between 1997 and 1999, 34 out of 38 cases (89.5\%) of MSF were reported in the provinces of Cuneo, Alessandria, and Torino (Fig. 1). The province of Cuneo was consistently characterised by the highest incidence and numbers of notified cases were above Poisson expectations in 1997 and 1998 (Table 1, Fig. 1).

\subsection{Spatial analysis}

In the study area, as evaluated by $K$-function and Monte Carlo simulation, examined dogs were clustered within distances of less than $2 \mathrm{~km}$ from residence sites of human cases of MSF (Figs. 2 and 3). Eighteen out of $116(15.5 \%)$ examined dogs were IFA+ (titre $\geq 1: 160$ ) for antibodies against $R$. conorii. Thirty-six $(31.0 \%)$ and $47(40.5 \%)$ dogs were IFA + at cut-off titres $\geq 1: 80$ and $\geq 1: 40$, respectively. Ten $(55.6 \%)$ of the highly IFA + dogs (titre $\geq 1: 160$ ) were clustered within $0.6 \mathrm{~km}$ from a MSF case (Fig. 2). Comparing $K$-functions for all dogs sampled with IFA+ dogs, clustering of seropositive dogs was significantly higher $(P<0.01)$ than that for all dogs at distances of 5.6 and $5.9 \mathrm{~km}$, as shown by results of

Table 1

Occurrence of human clinical cases of MSF in three provinces of Piemonte (1997-1999)

\begin{tabular}{llccc}
\hline Year & Province & Inhabitants & $\begin{array}{c}\text { Notified cases of } \\
\text { MSF (incidence }\end{array}$ & Probability $^{\mathrm{a}}$ \\
\hline 1997 & Cuneo & 554348 & $6(10.8)$ & 0.002 \\
& Alessandria & 433299 & $2(4.6)$ & 0.26 \\
& Torino & 2219971 & $2(0.90)$ & 0.97 \\
& Piemonte region (total) & 4291441 & $10(2.3)$ & - \\
1998 & Cuneo & 555444 & $7(12.6)$ & 0.002 \\
& Alessandria & 431988 & $1(2.3)$ & 0.73 \\
& Torino & 2216582 & $4(1.8)$ & 0.90 \\
& Piemonte region (total) & 4288051 & $13(3.0)$ & 0.14 \\
& Cuneo & 557430 & $4(7.2)$ & 0.19 \\
& Alessandria & 430983 & $3(7.0)$ & 0.88 \\
& Torino & 2214282 & $5(2.3)$ & - \\
\hline
\end{tabular}

\footnotetext{
${ }^{\mathrm{a}}$ Cases per one million inhabitants per year.

${ }^{b}$ Probability that the expected number of cases is greater than the observed number (Poisson distribution).
} 


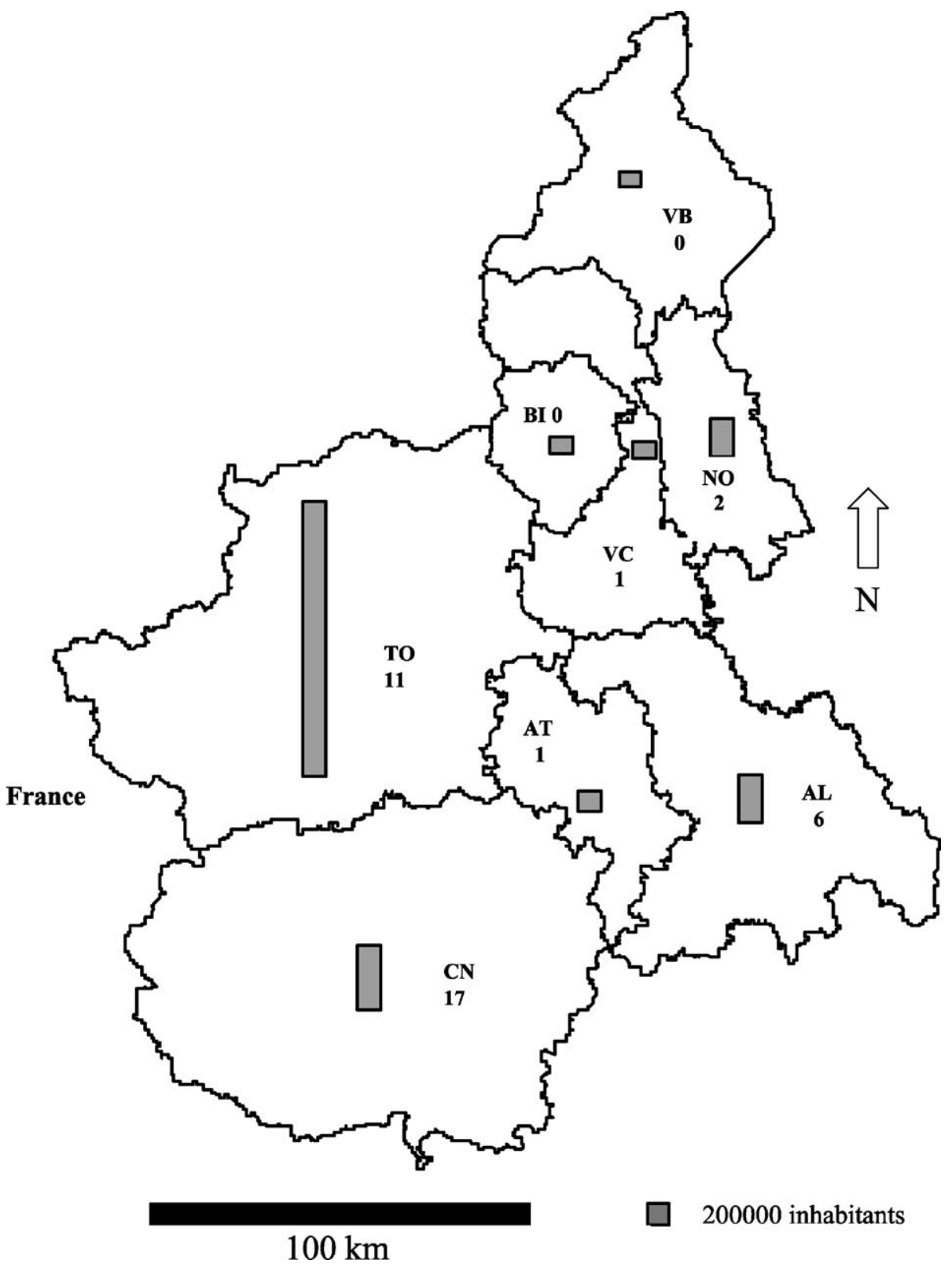

Fig. 1. Cases of MSF ( $n=38$ ) by province within the Piemonte region of Italy reported between 1997 and 1999 (TO: Torino; CN: Cuneo; AL: Alessandria; AT: Asti; VC: Vercelli; NO: Novara; BI: Biella; VB: Verbania). 


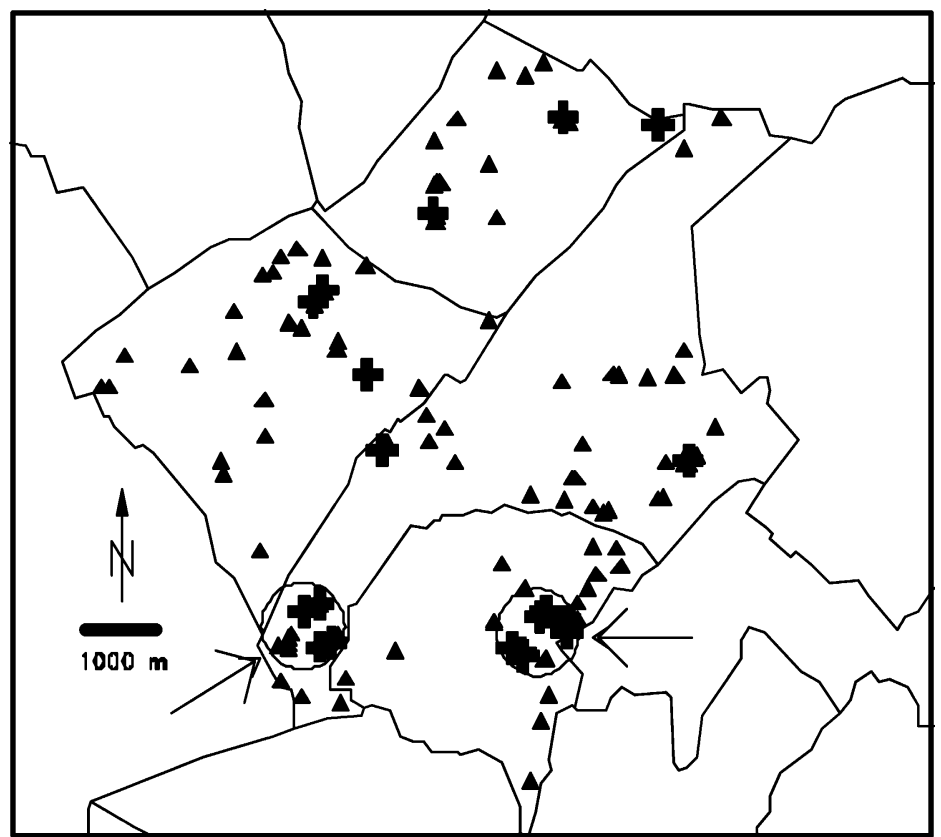

Fig. 2. Spatial distribution of $116 \mathrm{dogs}$ that were tested for antibodies against $R$. conorii in four municipalities in Cuneo province, Piemonte (1997-1999). Triangles represent dogs that were IFA-, crosses mark IFA+ dogs (titre $\geq 1: 160$ ). Arrows point to a $600 \mathrm{~m}$ radius drawn around the residences of two human cases of MSF.

permutation and bootstrap (Figs. 4 and 5-larger distances not shown in these figures). At distances of 2.0 and $5.3 \mathrm{~km}$, clustering verged on the threshold of significance $(P \sim 0.01)$ by permutation but was not significant by the bootstrap method. Eight IFA + dogs were at distances $>2.6 \mathrm{~km}$ from human cases of MSF and their spatial distribution showed no departure from randomness (data not shown in Figs. 4 and 5).

\subsection{Analysis of risk factors}

Some of the questionnaire variables (dogs' breed and age, distance from human cases of MSF, year of blood collection) were recorded based upon the following criteria. Eighty-five $(73.3 \%)$ out of 116 examined dogs were mixed breed while the remaining 31 belonged to 11 different breeds. It was therefore decided to divide dogs breeds into 'mixed' and 'other breeds'. Median (Q1-Q3) ages were 3.5 (2.0-5.0) and 2.0 (1-5) years in IFA+ and IFA - dogs respectively, and the difference between medians was not significant $(P=0.39$, Wilcoxon two-sample test). However, the prevalence of IFA+ dogs was $9.1 \%(n=33)$ and $9.5 \%(n=21)$ for $1-2$-year-old dogs, respectively, whereas it ranged from 26.7 to $33.3 \%$ in 3-6-year-old dogs $(n=43)$. No IFA+ animals were found in dogs older than 6 years $(n=16)$. Age was therefore coded to allow comparison of 3-6-year-old dogs with all other ages which were combined into one group. The relationship between distance from human 


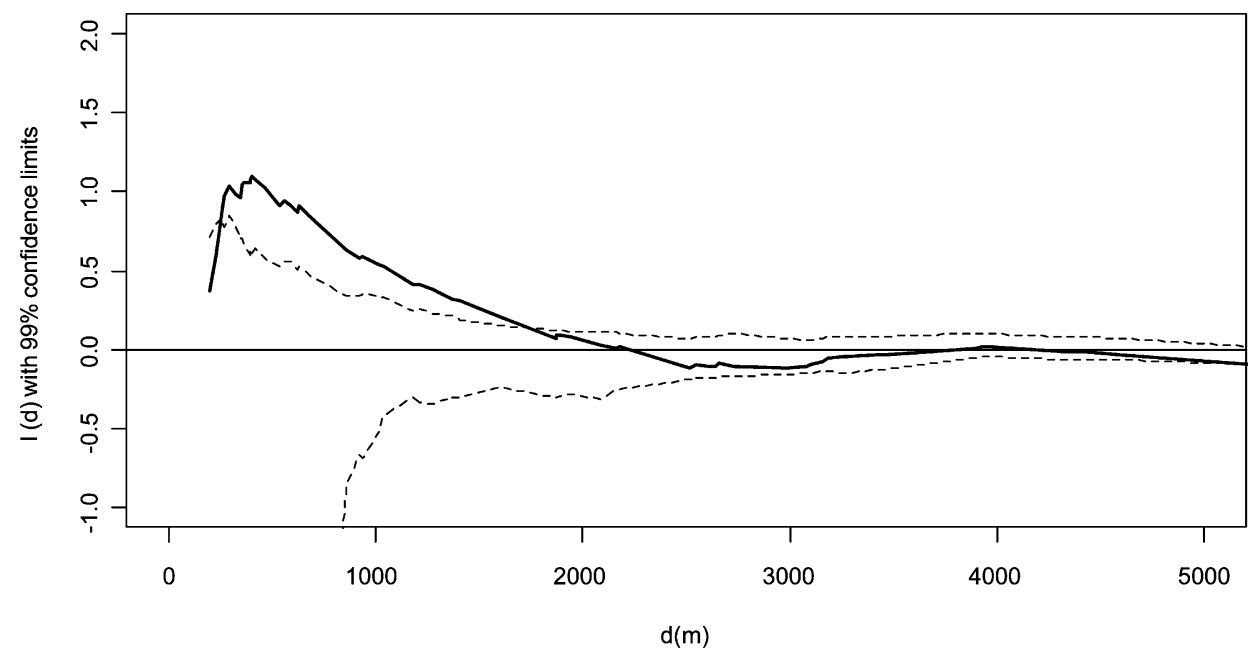

Fig. 3. Graphical comparison of estimated and theoretical $K$-function by $\hat{l}(d)$ (Eq. (2)) for 116 dogs that were examined for antibodies against $R$. conorii in Cuneo province (1997-1999). The bold line represents $\hat{l}(d)$ values as estimated by the data and dashed lines are upper and lower $99 \%$ CI as obtained by simulated sets of co-ordinated pairs within the study area. At distances where the estimated $\hat{l}(d)$ is greater than the upper CI, dogs were considered significantly clustered around residences of human cases of MSF. The horizontal line corresponds to a random distribution of dogs.

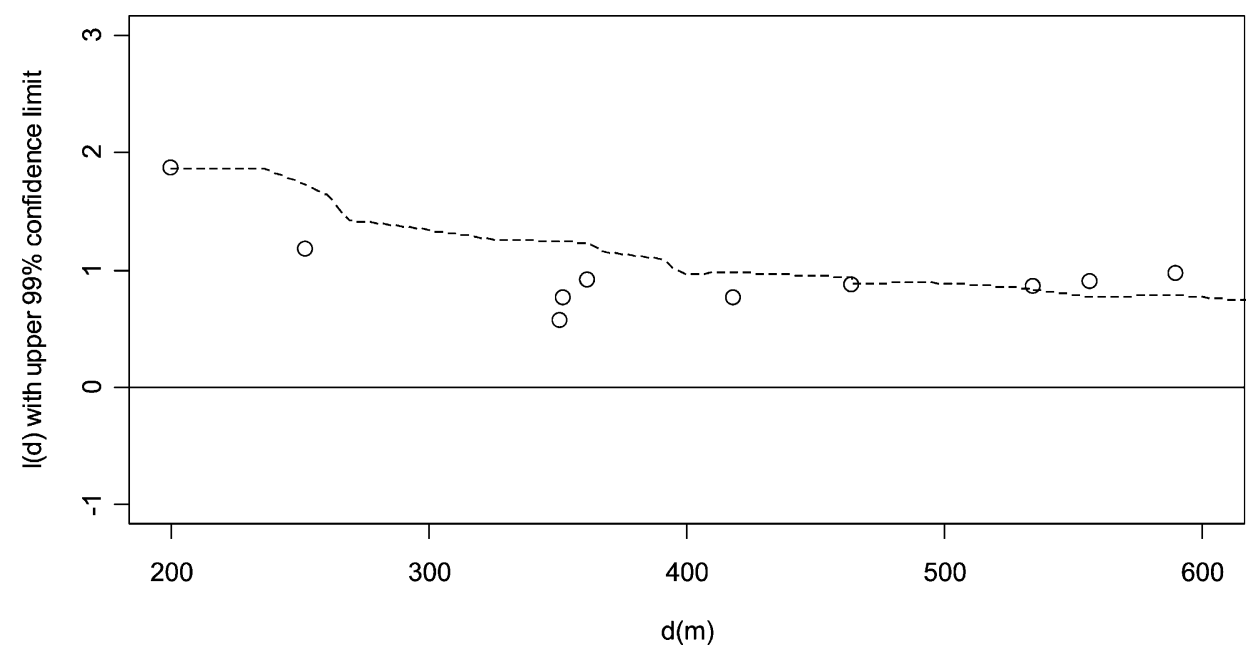

Fig. 4. Comparison of clustering of the 18 IFA+ dogs and all 116 dogs tested, around the residences of two human cases of MSF in the Cuneo province (first $0.6 \mathrm{~km}$ only presented). Circles represent comparison of $K$-functions by $\tilde{l}(d)$ (Eq. (3)) for observed data, whereas the dashed line represents the 99th percentile of values of $\tilde{l}(d)$ that were obtained by drawing 100 random permutations from the original data. At distances where the estimated $\tilde{l}(d)$ was greater than the 99th percentile, it was concluded that IFA+ dogs were significantly more clustered than for all $\operatorname{dog}$ tested. Minimum simulated values of $\tilde{l}(d)$ ranged from -0.12 to -15.6 but these are not shown. 


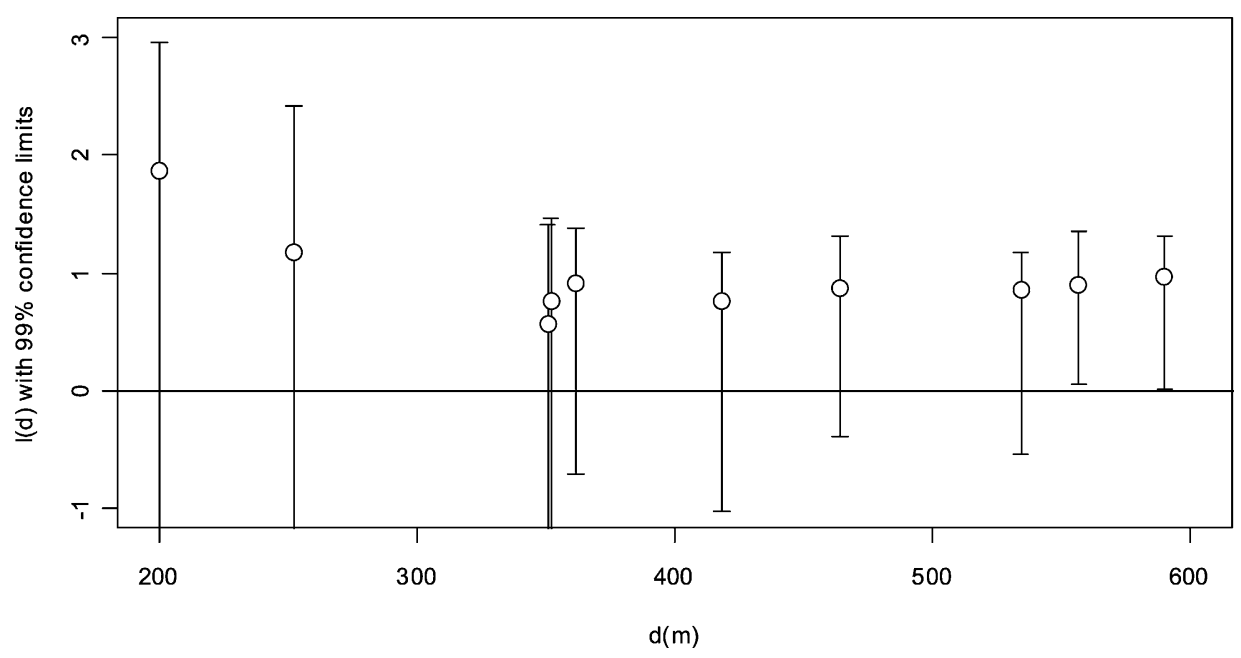

Fig. 5. Comparison of clustering of 18 IFA+ dogs and all 116 dogs tested, around residences of two human cases of MSF in the Cuneo province (first $0.6 \mathrm{~km}$ only presented). Error bars represent $99 \% \mathrm{CI}$ of $\tilde{l}(d)$ (Eq. (3)) that were obtained by bootstrap. At distances where lower limits were greater than zero, it was concluded that IFA+ dogs were significantly more clustered than for all dogs. Lower CI of the first four observations ranged from -12.5 to -15.0 and these are not shown.

MSF cases and IFA+ dogs was clearly non-linear. In fact, 10 of 29 dogs (34.5\%) living within distances less than the first quartile $(0.77 \mathrm{~km})$ were IFA+ while no positive dogs were found between $0.77 \mathrm{~km}(\mathrm{Q} 1)$ and $2.7 \mathrm{~km}(\mathrm{Q} 2)$. However, 3 of $29(10.3 \%)$ dogs were IFA+ between $2.7 \mathrm{~km}(\mathrm{Q} 2)$ and $4.4 \mathrm{~km}(\mathrm{Q} 3)$, and 5 dogs (17.2\%) were IFA+ positive at larger distances $(>4.4 \mathrm{~km})$. Dogs were therefore divided into those living within Q1 distance from a human MSF case and those living at longer distances. None of the 6 dogs that were examined in autumn 1997 were IFA+, whereas 8 dogs out of 54 (14.8\%) examined in 1998 and 10 out of $56(17.9 \%)$ in 1999 were IFA+. Explanation of coding of binary variables, and percentages of IFA+ in dogs at different exposure levels are reported in Table 2. Proximity, confinement, breed, treatments and age had a $\chi^{2}$ with $P<0.25$ in two-by-two table analyses and were therefore included as predictors in the first logistic regression model (likelihood ratio $\chi^{2}=29.8$, d.f. $=5, P<0.001$ ). Breed (Wald $\chi^{2}=0.84, P=0.36$ ), was subsequently excluded from the final model which contained the remaining three predictors. The final logistic regression analysis yielded a significant likelihood ratio $\left(\chi^{2}=29.0\right.$, d.f. $=4$, $P<0.001)$, and Hosmer and Lemeshow test showed no evidence of poor fit $\left(\chi^{2}=4.6\right.$, d.f. $=6, P=0.60)$. Odds ratios from bivariate and logistic regression analysis of questionnaire variables are reported in Table 2 . These analyses confirmed the spatial association between dogs' IFA+ status and clinical MSF in humans. Dogs between 3 and 6 years of age were most likely to be IFA+, as well as those that were previously treated for ectoparasites. Finally, there was a tendency towards a higher risk of being IFA+ in free-ranging dogs as compared to confined ones, although there was much uncertainty associated with such an effect. 
Table 2

Risk factor results for $R$. conorii by IFA (cut-off titre $\geq 1: 160$ ) in 116 dogs from four municipalities of Cuneo province (1997-1999) ${ }^{\mathrm{a}}$

\begin{tabular}{|c|c|c|c|c|}
\hline Risk factor & Code description & Code & $\begin{array}{l}\text { Prevalence } \\
(\%)\end{array}$ & $\begin{array}{l}\text { Odds ratio } \\
(95 \% \mathrm{CI})\end{array}$ \\
\hline \multirow[t]{2}{*}{ Proximity } & $\begin{array}{l}\text { Dog location within the first quartile }(0.77 \mathrm{~km}) \\
\text { of all dogs from a human MSF case }\end{array}$ & 1 & 34.5 & $12.6(3.0-52.8)$ \\
\hline & Otherwise & 0 & 9.2 & \\
\hline \multirow[t]{2}{*}{ Rural } & Owner resident in the countryside & 1 & 12.6 & $0.45(0.16-1.3)$ \\
\hline & Owner resident in a village & 0 & 24.1 & \\
\hline \multirow{2}{*}{$\begin{array}{l}\text { Confinement } \\
\qquad(n=112)\end{array}$} & Dog not confined & 1 & 18.1 & $9.6(0.73-127.0)$ \\
\hline & Dog confined to house, chained, or in a yard & 0 & 5.6 & \\
\hline \multirow[t]{2}{*}{ Other animals } & Other animals present at owner's residence & 1 & 13.2 & $0.66(0.24-1.8)$ \\
\hline & Otherwise & 0 & 18.8 & \\
\hline \multirow[t]{2}{*}{ Year } & Blood collected in 1999 & 1 & 17.9 & $1.4(0.51-3.9)$ \\
\hline & Blood collected in 1997 or 1998 & 0 & 13.3 & \\
\hline \multirow[t]{2}{*}{ Spring } & Blood collected in May or June & 1 & 19.4 & $1.5(0.50-4.3)$ \\
\hline & September, October, or November & 0 & 14.1 & \\
\hline \multirow[t]{2}{*}{ Use $(n=114)$} & Shepherd and hunting dogs & 1 & 20.8 & $1.6(0.50-4.9)$ \\
\hline & Companion dogs & 0 & 14.4 & \\
\hline \multirow[t]{2}{*}{ Ticks $(n=114)$} & $\begin{array}{l}\text { Ticks seen on dog by owner or at blood } \\
\text { collection }\end{array}$ & 1 & 13.2 & $0.73(0.24-2.2)$ \\
\hline & Otherwise & 0 & 17.1 & \\
\hline \multirow{2}{*}{$\begin{array}{l}\text { Treatments for } \\
\text { ectoparasites }\end{array}$} & Anti-parasitic sprays, powders or collars used & 1 & 22.2 & $5.7(1.2-26.7)$ \\
\hline & Otherwise & 0 & 8.3 & \\
\hline \multirow[t]{2}{*}{ Breed } & Mixed breed & 1 & 11.8 & $0.52(0.13-2.1)$ \\
\hline & Other breeds & 0 & 25.8 & \\
\hline \multirow[t]{2}{*}{ Sex } & Female & 1 & 12.8 & $1.4(0.45-4.2)$ \\
\hline & Male & 0 & 16.9 & \\
\hline \multirow[t]{2}{*}{ Age $(n=113)$} & 3-6-Year-old dogs & 1 & 30.2 & $7.3(1.9-28.4)$ \\
\hline & Other age $(<3$ or $>6$ years of age $)$ & 0 & 7.1 & \\
\hline
\end{tabular}

${ }^{a}$ Odds ratios were obtained using two-by-two table analysis or by multiple logistic regression (italics).

\section{Discussion}

In Piemonte, MSF is most frequently reported from the province of Cuneo, which is characterised by central plains (pastures or arable land) and accounts for 53\% of cattle and $70 \%$ of pigs of the region but only contains $13 \%$ of the human population (Anon., 1998). It has been previously suggested that the Italian rural and semi-rural conditions, such as those found in Cuneo, may favour the establishment of MSF foci (Maroli et al., 1996). Similar environmental conditions can be found in parts of the province of Alessandria (Piemonte) which has the second highest incidence of MSF in Piemonte. In this study, through the examination of $116 \mathrm{dogs}$ in a $61 \mathrm{~km}^{2}$ area, dogs that were IFA+ for $R$. conorii 
were spatially associated with residences of previously recorded human cases of MSF. The highest proportion of IFA+ dogs was found in the populations living in the same villages (Morozzo and Margarita) where the disease had been recorded in people.

Using the $K$-function, it was possible to detect clustering in the dogs that were sampled and to make comparisons between the IFA+ and IFA - groups. Monte Carlo and bootstrap simulations were used to assess the statistical significance of the observed clustering. Comparison of the degree of aggregation of using the two different simulation approaches demonstrated that there was significant clustering of IFA+ dogs at distances between 5 and $6 \mathrm{~km}$ from human cases of MSF. Results of bootstrap simulation, which was based on sampling with replacement from IFA+ dogs, were slightly more cautious than those obtained by permutation (sampling without replacement from all examined dogs) in assessing significant clustering of IFA+ dogs. Particularly, at the first distance-step $(\sim 0.2 \mathrm{~km})$, the highest value of $\tilde{l}(d)$ that was obtained by sampling without replacement corresponded to the observed value (Fig. 4). Conversely, sampling with replacement, the dog at $\sim 0.2 \mathrm{~km}$ could be randomly sampled more than once yielding the upper error bar in the bootstrap results (Fig. 5).

Classical epidemiological analysis by multiple logistic regression confirmed location as the most important risk factor associated with high IFA + titres (titre $\geq 1: 160$ ). The absence of an effect of rural habitat can be attributed to blurred or non-existent distinction between the rural and urban environment in this study area. The results suggested that the relatively high concentration of dogs that were relatively free to roam within the two villages increased the exposure to tick bites and favoured $R$. conorii transmission (Mumcuoglu et al., 1993; Raoult et al., 1993). Tick abundance was not evaluated as questionnaire data collection would probably not have been a valid method for gathering such information. However, tick abundance is generally associated with high canine density. Therefore, within rural areas of Cuneo, the intensity of $R$. conorii transmission and the risk of MSF were highest at locations where both human and canine population were concentrated. The apparent absence of year-to-year variations in dogs' IFA status suggested the presence of an established foci of $R$. conorii, whereas the seeming lack of seasonality could be explained by a continuous exposure to tick bites during the study period, or by a relatively long duration of dogs' antibody response. The highest IFA+ prevalence in 3-6-year-old dogs should be further investigated. Additional information should also be gathered on the treatment for ectoparasites in order to allow for a more detailed exploration of the positive association with IFA+ status of dogs. The effects of risk factors and spatial associations were weak when analyses were carried out using lower titres $(<1: 160)$ as cut-off values (results not shown). Specificity of the IFA test could be compromised by the use low cut-off values. Therefore, by using a high titre cut-off $(\geq 1: 160)$ in these analyses, the effects of risk factors from intense and specific exposure to $R$. conorii through tick bites were highlighted.

\section{Conclusions}

This study, carried out in a relatively small area, indicated that villages of the rural province of Cuneo are potential emergent foci of MSF in northern Italy. This type of study should be repeated for other MSF foci in Piemonte. Should these results be confirmed by 
other studies then canine serology could be profitably used as a sensitive indicator for the risk of MSF at the village/municipality level. The potential to provide MSF risk maps based on canine serology, would be a useful epidemiological tool from a public health perspective. Habitat characterisation, and the evaluation of natural and human related factors should be carried out to help explain spatial variations in the risk of MSF. Moreover, further studies should be directed to investigate the distribution and abundance of populations of potential hosts for immature stages of $R$. sanguineus, and competent reservoirs for $R$. conorii that may be critical for the intensity of transmission of the infection (Mumcuoglu et al., 1993).

\section{Acknowledgements}

We thank the personnel of AUSL 15, Cuneo, and of the municipalities involved in the study, who helped in the collection of blood samples from dogs. This study benefited from discussions with colleagues at the Society for Veterinary Epidemiology and Preventive Medicine 2001 Conference and at the First GISVET Conference. This work was supported by a grant to A. Mannelli by Regione Piemonte, Direction Public Health.

\section{References}

Anon., 1990. Using Atlas-GIS ${ }^{\circledR}$. Strategic Mapping Inc., Santa Clara, CA.

Anon., 1998. Annual Report. Piemonte Region, Direction of Public Health, Animal Health Section. http://www.regione.piemonte.it.

Boots, B.N., Getis, A. (Eds.), 1998. Point Pattern Analysis. Sage, Newbury Park, CA.

Dean, A.G., Dean, J.A., Coulombier, D., Brendel, K.A., Smith, D.C., Burton, A.H., Dicker, R.C., Sullivan, K., Fagan, R.F., Arner, T.G., 1995. Epi Info, Version 6: A Word Processing, Database and Statistics Program for Public Health on IBM-Compatible Microcomputers. Center for Disease Control and Prevention, Atlanta, GA.

Delgado, S., Carmenes, P., 1995. Canine seroprevalence of Rickettsia conorii infection (Mediterranean spotted fever) in Castilla y Leon (northwest Spain). Eur. J. Epidemiol. 11, 597-600.

Fotheringham, A., Brunsdon, C., Charlton, M. (Eds.), 2000. Quantitative Geography. Perspectives on Spatial Data Analysis. Sage, London.

Getis, A., Franklin, J., 1987. Second order neighborhood analysis of mapped point patterns. Ecology 68, $473-477$.

Hosmer, D.W., Lemeshow, S. (Eds.), 1989. Applied Logistic Regression. Wiley, New York, 307 pp.

Keysary, A., Torten, D.N., Gross, E.M., Torten, M., 1988. Prevalence of antibodies to Rickettsia conorii in dogs in Israel and its relation to outbreaks in man. Isr. J. Vet. Med. 44, 103-107.

Kitron, U., Jones, C.J., Bouseman, J.K., Nelson, J.A., Baumgarter, D.L., 1992. Spatial analysis of the distribution of Ixodes dammini (Acari: Ixodidae) on white-tailed deer in Ogle County, Illinois. J. Med. Entomol. 29, $259-266$.

La Scola, B., Raoult, D., 1997. Laboratory diagnosis of rickettsioses: current approaches to diagnosis of old and new rickettsial diseases. J. Clin. Microbiol. 35, 2715-2727.

Manly, B.F.J. (Ed.), 1991. Randomization and Monte Carlo Methods in Biology. Chapman \& Hall, London.

Maroli, M., Khoury, C., Frusteri, L., Manilla, G., 1996. Diffusione della zecca del cane (Rhipicephalus sanguineus Latreille, 1806) in Italia: un problema di salute pubblica. Ann. Ist. Super. Sanità 32, 387-397 (English Transl.).

Masoero, L., Dadone, R., Guercio, A., 1991. Rickettsia conorii: indagine sierologia sulla popolazione canina di un comune del Cuneese, in seguito ad un focolaio di febbre bottonosa. Il Nuovo Progresso Veterinario 21, 716-718 (English Transl.).

Mumcuoglu, K.Y., Frish, K., Sarov, B., Manor, E., Gross, E., Gat, Z., Galun, R., 1993. Ecological studies on the brown dog tick Rhipicephalus sanguineus (Acari: Ixodidae) in southern Israel and its relationship to spotted fever group rickettsiae. J. Med. Entomol. 30, 114-121. 
O’Brien, D.J., Kaneene, J.B., Getis, A., Lloyd, W., Rip, M.R., Leader, R.W., 1999. Spatial and temporal distribution of selected canine cancers in Michigan, USA, 1964-1994. Prev. Vet. Med. 42, 1-15.

Raoult, D., Roux, V., 1997. Rickettsioses as paradigms of new or emerging infectious diseases. Clin. Microbiol. Rev. 10, 694-719.

Raoult, D.H., Tissot-Dupont, P., Caraco, P., Brouqui, M., Drancourt, M., Charrel, C., 1992. Mediterranean spotted fever in Marseille: descriptive epidemiology and the influence climatic factors. Eur. J. Epidemiol. 8, 192-197.

Raoult, D.H., Tissot-Dupont, C., Chicheportiche, O., Peter, B., Gilot, B., Drancourt, M., 1993. Mediterranean spotted fever in Marseille, France: correlation between prevalence of hospitalized patients, seroepidemiology, and prevalence of infected ticks in three different areas. Am. J. Trop. Med. Hyg. 48, 249-256.

SAS, 1990a. SAS ${ }^{\circledR}$ Language: Reference, Version 6, 1st ed. SAS Institute Inc., Cary, NC.

SAS, 1990b. SAS ${ }^{\circledR}$ Guide to Macroprocessing, Version 6, 2nd ed. SAS Institute Inc., Cary, NC.

SAS, 1996. SAS/STAT ${ }^{\circledR}$ Software: Changes and Enhancements through Release 6.11. SAS Institute Inc., Cary, NC.

Segura-Porta, F., Diestre-Ortin, G., Ortuno-Romero, A., Sanfeliu-Sala, I., Font-Creus, B., Munoz-Espin, T., de Antonio, E.M., Casal-Fabrega, J., 1998. Prevalence of antibodies to spotted fever group rickettsiae in human beings and dogs from an endemic area of Mediterranean spotted fever in Catalonia, Spain. Eur. J. Epidemiol. 14, 395-398. 